\title{
Open Access Journal of Microbiology \& Biotechnology
}

\section{The Growing Distrust of Science}

\section{Robin PE* $^{*}$}

Marywood University, Scranton, PA, USA

*Corresponding author: Robin P Ertl, PhD in Biochemistry, Marywood University, Scranton, PA, USA; Email: rertl@maryu.marywood.edu

\section{Editorial}

If it is the job of science to elucidate the processes of nature and use that knowledge to solve problems, why do more and more people distrust science? People from all walks of life have taken stands against science on a variety of issues including: Climate Change, Oceanacidification, Vaccinations, Environmental regulation, Evolution, Medicine and Nutrition. The distrust of science is nothing new, when Galileo indicated that the earth circled the sun, he was declared a heretic and placed under house arrest for the rest of his life. Similarly, Darwin's theory of evolution came out over 150 years ago and is a corner stone of modern biology, yet it is still being rejected by knowledgeable people and the teaching of evolution challenged in certain states. Some propose that people cling to what they know, and that changing entrenched opinion can be a slow process for both individuals and for society.

It is easy to suggest that the fault lies with people not considering all the facts and just wanting the solution. Scientists, though, are also part of the problem when they don't explain the confidence that can be given to a study or the process of eliminating possibilities. Scientific discovery starts from tying to explain an observation. From an observation a hypothesis is created. The hypothesis is tested and either supported or rejected. This process is repeated until there is a clear enough understanding to draw a conclusion. As more data becomes available, each new refined interpretation needs to explain all of the previous data. Thus, equal weight is not given to an observation with little background, as a study that builds upon the data of multiple investigations. That is to say whether an interpretation is correct increases with the number of explained observations. The public generally does not understand where a study fits into this process and how much confidence to give its interpretation. The field of science fosters this problem by trying to sell the importance of each article.

Epidemiological studies presented in the media are seen by the public as fact, rather than the first step in the process of discovery. These studies observe trends on a background where multiple variables are changing. As these trends are explored the actual cause may be different than first explained. As the public tries to make decisions based on these studies, it loses confidence each time the explanation changes. A real world example is the epidemiological studies that consistently find that people who eat broccoli live longer [1]. It is noted that compared to other vegetables, broccoli has higher levels of antioxidants and other chemicals that can reduce the risk of cancer that can shorten lifespan. However, this trend has not been found in controlled animal studies examining broccoli. It can always be that lab animals are different than humans, but it may also be that humans who eat broccoli are also eating and living healthier in other ways not controlled by the study. To the public, it appears that one day it is good for you and the next day it is bad.

Science does not withhold information until the end as refining knowledge is an ongoing process. Unfortunately, each article tries to indicate its importance by seeming to have insight or the solution. In trying to sell the importance of the article it is easy to present one side of an argument and only mention in passing qualifiers or alternate explanations, because less in known about them. However, the assumptions and other possibilities can alter the conclusions. Part of the problem is that we have been taught to present our interpretations in the best possible light to get grants or to increase the significance of a publication. Rarely, though, is the truth as simple and straight forward as initially presented. This leads to the impression that the science is not reliable or flawed. Is it then surprising that the public is suspicious of GMOs, 


\section{Open Access Journal of Microbiology \& Biotechnology}

especially given that some of the first GMOs had problems (ex. peanut allergies, increasing toxic natural pesticides)?

Even if the weakness of the study is indicated, how it is phrased and where it is noted can be missed by people not in the field. There was the observation that women eating soybean products had fewer post-menopausal symptoms. In a journal article [2], the authors tested the hypothesis that the isoflavones might be acting as estrogen mimics. The study did not find any affect by isoflavones, but in the discussion noted that they only tested isoflavones and there might be interactions with other components of soy beans. Network news picked up on the article and reported that soy is not good for menopausal hot flashes or bone loss. The news media possibly only read the abstract or did not understand the inference that other components of soy beans might induce enzymes like P450 that could activate the isoflavones. These contradictions cause the public to see the science as unreliable, rather than the study was merely a step in the discovery process. Similar confusion is seen in studies of dietary cholesterol or the efficacy of PSA tests. If abstracts are used to sell the importance of the article not detract, it becomes easy to see how people outside the field merely reading the more accessible portions of the article could miss the qualifiers mentioned in the technical discussion. Similar confusion is found in press coverage of conferences. By the very nature of conferences, scientists often present data that is in process in an effort to raise awareness and seek discussion. The data presented is generally not peer reviewed and a significant number will never be published in the current form. The media has presented some of these studies as cutting edge fact without considering where this is in the discovery process, and thus, the weight that should be given to the conclusions.

One of the problems is that it is hard to summarize a complex argument quickly. Thousands of articles are written in science each month but those that have the greatest knowledge in the field often fail to summarize the findings in a balanced way that the public can understand and use. Thus, it is left up to the news media and other people with less knowledge of the intricacies to interpret the research. When the implementation of scientific discoveries create problems it engenders a lack of public trust. Much of science requires a great deal of investment in time, and money. Investors want to recoup that money with interest as quickly as possible, thus there is pressure to bring technology to market before the consequences are fully understood. Proper disposal of waste from nuclear fission reactors, the long-term consequences of the Chernobyl and Fukushima Daiichi disasters, the problems created by the use of DDT and mesothelioma caused by exposure to asbestos are examples of exploited discoveries that had dire consequences that then needed to be solved. The pressure to increase profits has also created several scandals in the pharmaceutical industry where knowledge of deleterious side effects has been suppressed.

The DuPont PFOA scandal, where there were indication of problems as earlier as the 1960's but where PFOA was only pulled from the market in 2013 calls into question the ethics of who knew what when, and whether scientists have a moral obligation to disclose toxicity. Industry has made a lot of money off innovations in science. However, some industries resist interference that limits profit when scientific studies warn of danger. The public generally assumes that if it is available it must be safe and there is a loss of confidence when it is determined that a hazard exists. In our system, full explanations are rarely provided. For example, a plastic frozen dinner tray that says microwave safe is assumed to be safe to cook food. In reality it only indicates that it won't melt, not whether it is leaching plasticizers into the food.

There is also pressure in the ivy covered towers of academia, where the moto is publish or perish. Given the need for publications to be promoted or obtain tenure, there is tremendous pressure to circumvent the rules. Beyond the scandals of fabricated data, pushing data to merely get publications can add confusion to a field of study and cause a loss of confidence when the full story is revealed. The public expects science to be reliable and knowledgeable, thus when the unpredicted happens it lays the foundation for the lack of trust. A while ago the U.S. Forest service changed its policy to allow limited burns as necessary for forest development. However, since the forests were not allowed to burn in quite some time, the first fires got out of control, calling the science into question. Perception is also a big part of the trust issue. Even if there was no significant consequence, the use of mercury in vaccines in the latter half of last century did not help public trust, as mercury used in the milliner industry was long known to affect cognition and memory (mad as a hatter). This brought up a lot of public questions about the potential links between childhood vaccinations and proper neural development that still persist.

The resistance to scientific evidence is exacerbated when it is politicized. Politicians can take advantage of science or the distrust of science depending on the perceived need. In 2015 the U.S. House of Representatives passed HR 185 in an attempt to limit the scope of the EPA to enforce the clean water act. The motivation appears to 


\section{Open Access Journal of Microbiology \& Biotechnology}

be a concern over how enforcement might impact the economy rather than protecting the environment. Yet the U.S. Congress used science when the EPA failed to take more significant action in the Flint, Michigan to protect the water supply. When authorities can ignore scientific evidence and concerns it directly influences the public perception of whether science can be trusted. The underlying motives in politics are at the heart of the debate over climate change.

As the consequences of human activity and disease become greater and greater the public needs to trust science in order to make sound decisions in a timely fashion. If society refuses to consider scientific warnings, it may affect human existence on the planet. This seems extreme but nuclear disasters, climate change, environmental pollution, alterations of the human genome can have far reaching effects. It is thus beholden on science to resist the pressures to make more out of the data than what it indicates. Science needs to make the evidence clear to the public and indicate how much weight can be given to any piece of data and how it fits into the overall picture.

\section{References}

1. Verhoeven DTH, Goldbohm RA, van Poppel G, Verhagen H, van den Brandt PA (1996) Epidemiological Studies on Brassica Vegetables and Cancer Risk. Cancer Epidemiology Biomarkers \& Prevention 5(9): 733-748.

2. Levis S, Strickman Stein N, Ganjei Azar P, Xu P, Doerge DR, et al. (2011) Soy Isoflavones in the Prevention of Menopausal Bone Loss and Menopausal Symptoms. Arch Intern Med 171(15): 1363-1369.

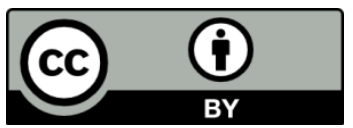

